\title{
LA EMOCIONALIDAD EN LAS ESTRATEGIAS DE MERCADEO: CASO INDUSTRIA FUNERARIA ${ }^{1}$
}

\section{EMOTIONALITY IN MARKETING STRATEGIES: CASE STUDY FUNERAL INDUSTRY}

\section{A EMOTIVIDADE NAS ESTRATÉGIAS DE MARKETING: CASO INDÚSTRIA FUNERÁRIA}

Duván Emilio Ramírez Ospina. ${ }^{2}$

Carolina Duarte Pareja ${ }^{3}$

Lina María Toro Gonzales ${ }^{4}$

\section{FORMA DE CITACIÓN}

Ramírez, DE., Duarte, C. \& Toro, LM. (2016). La emocionalidad en las estrategias de mercadeo: caso industria funeraria. Dimensión Empresarial, 14(1), 109-122

JEL: M31

DOI: http://dx.doi.org/10.15665/rde.v14i1.570

\section{RESUMEN}

En este escrito se analiza el manejo de la emocionalidad en las estrategias de mercadeo en empresas del sector funerario, destacando el carácter contextual y ambiental del marketing dentro de la cultura como base permeable de la sociedad, sus hábitos, costumbres y rituales. Se partió de un estudio cualitativo usando entrevistas en profundidad y observación; se hizo un acercamiento teórico al marketing emocional y se muestran las relaciones encontradas entre este y las estrategias de mercadeo que están siendo usadas por las empresas funerarias. Identificando el manejo de la emocionalidad implementado en el sector funerario por medio de "talleres de duelo"; eventos post-mortem de carácter masivo, donde los dolientes asisten a charlas programadas cada tres meses. Este tipo de eventos por su periodicidad, asisten un número elevado de personas, dificultando que la experiencia sea individual, detallada y que facilite un contacto directo entre el cliente y la empresa.

Palabras clave: Industria funeraria, emocionalidad, marketing, marketing emocional, planeación estratégica.

1 Artículo de investigación, resultado del trabajo "Relación entre las estrategias de mercadeo y el manejo dela emocionalidad en la industria funeraria", elaborado como requisito parcial para optar el título de Magister en Mercadeo de la Universidad de Manizales, www.umanizales.edu.co. El proceso investigativo se inició en julio de 2013 y finalizó en agosto de 2015. Fecha de recepción 16/11/2'15. Fecha de aceptación 10/12/2015.

2 Doctor en Administración; Magister en Gerencia del Talento Humano; Especialista en Mercadeo; Economista; Decano Facultad de Ciencias Contables, Económicas y Administrativas de la Universidad de Manizales. dramirez12@unisimonbolivar.edu.co Profesor de Posgrados Universidad Simón Bolivar; Publicaciones: Capital Humano: Una visión desde la teoría crítica. Cadernos Ebape.Vol.13.No 2. P.p 315 - 331. La imagen y la Expectativa Frente a la Realidad de Villavicencio como Destino Turístico, Revista Orinoquía. Vol.18. No 1. Pag. 112 - 127. Comportamiento del Consumidor Turístico. Revista Criterio Libre. Vol. 12. No 20. Enero Junio. Pág. 165 183.

3 Magister en Mercadeo; Especialista en Docencia Universitaria; Economista; Investigadora Universidad de Manizales. Karol77duarte@gmail.com

4 Magister en Mercadeo; Diseñadora Industrial; Investigadora Universidad de Manizales. Linis56@gmail.com Itorogo@gmail.com 


\section{ABSTRACT}

In this paper, it is analyzed the handling of emotions within marketing strategies in the funeral industry companies. There are highlighted items such as the contextual character of marketing within the culture as a permeable base of society, their habits, customs and rituals. It began with a qualitative study using in-depth interviews and observation. There was a theoretical approach to emotional marketing and the relationships found between them and the marketing strategies used by the studied undertakers. It was identified that the management of emotionality implemented in the funeral industry consisted on "mourning workshops"; post-mortem events of mass character, where mourners attend talks scheduled every three months. Due to their frequency, a large number of people attended these events, making difficult the individual, detailed and direct contact between the customer and the company.

Keywords: Funeral Industry, emotionality, marketing, emotional marketing, strategic planning.

\section{RESUMO}

Este artigo analisa o manejo da emotividade nas estratégias de marketing das empresas do setor funerário, destacando o caráter contextual e ambiental do marketing dentro da cultura como base permeável da sociedade, seus hábitos, costumes e rituais. Partiu-se de um estudo qualitativo, utilizando entrevistas em profundidade e observação; realizou-se uma abordagem teórica com o marketing emocional e mostram-se as relações encontradas entre este e as estratégias de marketing que estão sendo utilizadas pelas empresas funerárias. Identificando o manejo da emotividade implementada no setor funerário através de "oficinas de luto"; eventos "post-mortem" de caráter de massa, onde os enlutados comparecem a palestras agendadas a cada três meses. Estes tipos de eventos, por sua periodicidade, contam com a presença de um grande número de pessoas, dificultando que a experiência seja individual, detalhada e que facilite um contato direto entre o cliente e a empresa.

Palavras-chave: Indústria funerária, emotividade, marketing, marketing emocional, planejamento estratégico.

\section{INTRODUCCIÓN}

Este escrito es un resultado parcial de la Investigación "Relación entre las estrategias de mercadeo y el manejo de la emocionalidad en la industria funeraria", elaborada como requisito parcial para optar al título de Magister en Mercadeo de la Universidad de Manizales, por parte de dos de las autoras; en la cual se pretende establecer el papel desempeñado por el manejo de la emocionalidad en las estrategias de mercadeo de estas organizaciones; para el efecto, se hace una descripción de la importancia que tiene el marketing emocional en las estrategias orientadas a la fidelización de los clientes y al mejoramiento y humanización del servicio en la Industria funeraria como factor clave para la permanencia en el mercado.

En las empresas observadas se pudo establecer que el único factor diferenciador es "precio pagado vs servicio obtenido", sin considerar la realidad del consumidor que busca experiencias y no características tangibles de un producto o servicio, y esta experiencia solo se logra humanizando el servicio para crear un vínculo emocional entre el consumidor y la empresa, lo que genera preferencia por parte del cliente, en caso de requerir nuevamente sus servicios, o la recomiende a otros.

También se observó, que en general, estas organizaciones no cuentan con un departamento de mercadeo; por lo que el proceso de marketing está bajo la responsabilidad de los departamentos de ventas lo que lleva a prestar un servicio derivado de un acuerdo comercial suscrito entre las partes, un servicio a cambio de una cantidad de dinero, que no aplica los conceptos del mercadeo, por tanto no considera la situación particular por la que pasan los clientes cuando hacen uso de sus servicios. Por lo cual, es habitual que los clientes se quejen ante la empresa por presuntos incumplimientos entre lo pactado al momento de adquirir el plan exequial y lo recibido al momento del evento. 
Se encontró que lo más cercano al manejo de la emocionalidad que se está implementando en el sector funerario son los llamados "talleres de duelo", que suelen ser eventos post-mortem de carácter masivo, donde los dolientes asisten a charlas que suelen programarse cada tres meses. Este tipo de eventos tienen en contra que debido a la periodicidad con que se programan asisten un número elevado de personas, dificultando que la experiencia sea individual, detallada y que facilite un contacto directo entre el cliente y la empresa; además, para quienes han vivido la situación al inicio del trimestre ya puede ser muy tarde el taller.

En este caso, no se tiene en cuenta por parte de las empresas que la necesidad de recurrir a la asesoría profesional por parte del doliente puede llegar a ser inmediata, teniendo este que esperar hasta que se efectué el taller según la programación de la empresa, por tanto la programación está determinada más por aspectos propios de la racionalidad económica y no por las necesidades del cliente, constituyéndose una práctica de orientación a las ventas en perjuicio de una orientación al mercado.

Adicionalmente, es posible que el doliente no pueda asistir ya que al ser un evento masivo es posible que no se adecue a su horario por lo que en caso que definitivamente requiera del acompañamiento profesional, deberá pagar un dinero adicional, lo que hace que la empresa se convierta en un intermediario, haciendo que lo inicialmente planteado como una oferta de valor se transforme en otra transacción comercial de asistencia sicológica. También se encontró que solo una empresa de las observadas, está implementando cambios de nivel arquitectónico para generar experiencias sensoriales: salas más iluminadas sin colores lúgubres, espacios más abiertos, sonido y video en la sala que recuerden momentos de la vida del difunto; todo esto basado en el marketing experiencial.

Desde la perspectiva metodológica, se trata de una investigación de tipo cualitativo con un enfoque hermenéutico, la cual se realizó tomando como muestra las principales funerarias con cobertura nacional y que tienen sedes o sucursales en las ciudades de Manizales y Armenia. Para la recolección de in- formación se entrevistaron directivos y asesores comerciales de dichas empresas cuyas respuestas fueron categorizadas y relacionadas con ayuda del AtlasTi para posteriormente ser analizadas, a partir de esto se llega a las conclusiones.

De la misma forma, se hizo un proceso de observación en los sitios de prestación del servicio con el fin de establecer una relación entre lo que plantean los gerentes y ejecutivos comerciales de las empresas y la forma como se presta el servicio en la práctica, lo que constituye un proceso de triangulación que permite evidenciar que tan lejos o cerca se encuentra el planteamiento teórico del hacer práctico.

\section{MARCO TEÓRICO:}

\subsection{Marketing Emocional.}

Los primeros acercamientos teóricos al marketing emocional se originan a principios de los años ochenta del siglo XX, cuando Holbrook \& Hirschman (1982), resaltan el papel de las emociones como elemento clave del comportamiento de los consumidores, cuestionando los planteamientos de la teoría del mercadeo hasta ese momento, en el sentido que la toma de decisiones del consumidor sea un proceso de decisiones basado exclusivamente en una serie de argumentos racionales; por tanto, los autores destacan la necesidad de analizar y estudiar el papel de las experiencias que el consumidor desarrolla con la compra y uso del producto en su proceso de decisión.

En este orden de ideas, Hirschman (1999), plantea que el consumidor, de cualquier producto, tiene un rango emocional que puede moverse desde extrema felicidad a extrema tristeza, esta oscilación depende de las experiencias y el comportamiento de consumo de las personas, frente a una misma situación y responde a sus estados emocionales, los cuales tienen variaciones dependiendo de patrones culturales, de personalidad y demográficos preexistentes como la edad, la raza, el género o la cultura, entre otros.

En los comportamientos de consumo, se identifican dos que están completamente ligados a la emocionalidad del consu- 
midor: 1) Consumo impulsivo: Las compras impulsivas están asociadas a estados de ánimo positivos en niveles intensos o altos de felicidad, optimismo, entusiasmo entre otros; 2) Consumo compulsivo: Se refiere a compras que se hacen de forma repetitiva que destruye al consumidor de manera psicológica y financiera, pero lo provee de momentos en los que se libera la tensión, la ansiedad o la tristeza. (Hirschman, 1999).

Este planteamiento toma fuerza a finales de los años noventa del siglo XX, cuando Pine \& Gilmore (1998) formulan su teoría denominada "La economía de la experiencia" y Schmitt (1999) plantea el concepto de "Marketing experiencial". La economía de la experiencia, se basa en las nuevas características del consumidor, centrado en la búsqueda y experimentación de una serie de sensaciones, recuerdos y momentos calificados como extraordinarios y memorables. (Pine \& Gilmore, 1998).

Las experiencias son sucesos que involucran a los individuos de forma personal, presentándolas como una nueva fuente de valor de los bienes y servicios que es necesario añadir, dando lugar al desarrollo de lo que se denomina como progresión económica de la cadena de valor. Así, se incorpora al producto una serie de recuerdos o emociones, siendo éstas el origen de la percepción de una experiencia irrepetible que permitirá aumentar el valor del producto o servicio adquirido por el cliente, otorgándole, además, un carácter único y diferenciador frente a la competencia. (Moral \& Fernadez, 2011)

De igual manera, Pine \& Gilmore (1998) identifican los siguientes cuatro tipos de experiencia y participación del cliente: 1) Entretenimiento (Entertainment). Se trata del tipo de experiencia que una gran parte de las personas asocia con el entretenimiento. Se produce cuando se realiza una absorción pasiva de las experiencias a través de los sentidos; 2) Educativo (Educational). Una experiencia educativa implica la participación activa del sujeto involucrando su mente, sintiéndose atraído por el deseo de aprender y ampliar sus conocimientos; 3) Escapista (Escapist). El sujeto participa muy activamente en la actividad encontrándose totalmente inmerso en la misma. Algunos ejemplos de actividades escapistas son los parques de atracciones, la realidad virtual, la práctica de deportes, entre otros; 4) Estética (Esthetic). Una experiencia estética implica la observación y disfrute del entorno o del ambiente físico del lugar. De este modo, el atractivo físico es el factor determinante de la visita del lugar, por ejemplo, un museo, un entorno natural. Por consiguiente se puede deducir que las emociones percibidas por las personas en los diferentes momentos de consumo, varían dependiendo de si se es protagonista o espectador, y de qué tipo de experiencia se vive.

De la misma manera, Caro J \& Piñeros (2014), plantean que las experiencias de consumo pueden ser positivas o negativas y estas influyen en el comportamiento del consumidor; lo que implica la participación de las emociones en la formación y cambio de actitudes; el argumento básico de estos autores, es que algunas emociones que han llevado a la formación de las actitudes iniciales pueden hacer que estas sean más resistentes al cambio en caso de ambivalencia.

En su trabajo Caro J \& Piñeros (2014) observaron que las actitu $\neg$ des basadas inicialmente en experiencias negativas son más resistentes al cambio que las basadas en experiencias posi-tivas. Además, plantean que las experien $\neg$ cias negativas tienen una carga emocional mucho más fuer $\neg$ te que las positivas, donde predomina más el aspecto cog $\neg$ nitivo las emociones negativas constituyen estados más intensos que las emociones positi $\neg$ vas, por lo que conducen a acciones más concretas que las emociones positivas y esto hace que la actitud inicial formada a partir de emocio $\neg$ nes negativas del individuo sea más resistente al cambio que aquella actitud inicial formada a partir de emociones positivas.

Las emociones son muy específicas, esto considerando que se orientan hacia los factores que las originan, y se pueden clasificar de diferente manera, en función del tiempo se puede hablar de emociones vinculadas con el pasado que se manifiestan en el orgullo, o las vinculadas con el presente cuya manifestación se da por medio de la felicidad y las del futuro general esperanza; sin embargo, la clasificación más generalizada las agrupa en función de si son positivas o negativas. (Winterich \& Haws, 2011) 


\subsection{El Marketing Experiencial}

El concepto de Marketing Experiencial fue usado inicialmente por Betnd Schmitt (1999), quien define sus cuatro principales características en los siguientes términos:

A. El marco central es la experiencia del cliente. A diferencia del Marketing Tradicional, basado en las características funcionales del producto; el Marketing Emocional considera que las experiencias del cliente son el resultado de los estímulos provocados por los sentidos o por la mente del cliente en determinadas situaciones que permiten conectar la empresa y la marca con la forma de vida del cliente. De este modo, las experiencias aportan valores emocionales, cognitivos o sensoriales sustituyendo a los tradicionales valores funcionales del producto, lo que determina la actitud del consumidor hacia el producto.

Por lo tanto, el marketing experiencial centra su atención en las vivencias de los consumidores y sus sentimientos involucrados en el proceso de consumo; estas experiencias tienen sensaciones, emociones, conductas cognitivas, y dimensiones relacionales, no sólo funcionales; el objetivo es que el consumidor se comporte de acuerdo con sus impulsos y emociones, más que con su razón (Barrios, 2012).

En su concepción más actual, las actitudes hacia un producto o servicio pueden estar ba-sadas tanto en cogniciones como en aspectos de carácter más afectivo, en este sentido Clarkson, Tormala \& Rucker, (2011), indican que las cogniciones son pensamientos y creencias que el individuo ha generado durante el proceso de consumo y constituyen la parte más racional de la formación del juicio, el aspecto afectivo de la formación de una actitud respecto de un bien o servicio, está determinada por las emociones que experimenta el individuo como resultante del acto de consumo.

B. Los clientes son individuos racionales y emocionales. Los individuos son considerados sujetos que basan sus decisiones en elementos racionales, pero también, a menudo, en las emociones, las cuales despiertan una experiencia de consumo. Por ello, desde el Marketing Emocional se destaca la necesidad de adoptar una visión del cliente como un individuo no solamente racional sino como un sujeto que desea recibir estímulos y experiencias cargadas de emotividad y creatividad en su relación con el producto y la empresa. Según Jiménez B \& Martínez C (2016), el Marketing Experiencial es una disciplina que propone un tipo de comunicación bilateral con el consumidor, que considera que sus elecciones en el mercado son racionales y emocionales; por tanto, no se centran únicamente en las características funcionales de un producto o servicio, sino que valoran también la experiencia placentera que les genera conocerlos, adquirirlos o consumirlos.

C. Examen de la situación de consumo. El Marketing Emocional considera que el cliente no evalúa el producto analizando exclusivamente sus características y beneficios funcionales, sino que, además, estudia cuáles son las experiencias que le aporta en función de la situación de consumo en la que se ha utilizado. Las experiencias vividas por el cliente durante el consumo, son clave para lograr una mayor satisfacción y lealtad en el cliente, García Bobadilla (2010).

De acuerdo con lo anterior, según Caridad, Castellano \& Hernández (2015) consideran necesario ofrecer produc $\neg$ tos atractivos o satisfactorios y que además tengan un valor adicional basado en aspectos emocionales-vivenciales, que hagan la di $\neg$ ferencia con los competidores; para generar valor, transmi $\neg$ tiendo cercanía, fidelidad, y lealtad entre el cliente y el proveedor, para generar experiencias de consumo que se queden en la mente del consumidor.

Además, el examen de la situación de consumo considera no sólo el concepto de una categoría de producto, sino también, el significado dentro de una situación específica de consumo en un contexto socio-cultural más diverso. Por tanto, esta óptica del marketing se aleja de la visión del producto como un elemento aislado de consumo para pasar a considerarlo dentro de un contexto más amplio determinado por las características y peculiaridades de su situación de consumo, dando lugar al denominado "vector sociocultural de consumo" (VSCC). Por ello, 
el examen de situación o momento de consumo es fundamental para asegurar la correcta adaptación del producto a las necesidades y expectativas del cliente. (Moral \& Fernadez, 2011)

D. Métodos y herramientas ecléticos. El Marketing Emocional no se encuentra circunscrito a una metodología de investigación concreta sino que adopta instrumentos amplios y diversos. La idea original de esta teoría, gira en torno a lo que se denomina "Customer Experience", y considera esas experiencias de consumo como el resultado de las interacciones entre el cliente y un producto, la empresa o algún elemento de la misma, que genera una reacción positiva o no para el individuo. Moral \& Fernadez, (2011) consideran que se trata de una experiencia personal, e implica la participación del individuo, llevándose a cabo una evaluación de dicha experiencia a través de la comparación de las expectativas del cliente y de los estímulos recibidos.

En este orden de ideas, la propuesta de valor debe tener en cuenta un relato que enfatiza la importancia de las emociones, puesto que estas entretejen las redes que dan soporte al mercado ofreciendo un sentimiento de pertenencia y diferenciación a los clientes que es deseado por estos. La incorporación de las emociones y experiencias a las estrategias de negocio, así como la apelación a unas conductas y decisiones producidas por la interacción entre emoción y racionalidad, es un hecho presente en las relaciones entre consumidores y productos (Casani, Rodríguez-Pomeda, \& Sánchez, 2012)

A lo largo de la vida, todas las personas recopilan un conjunto de experiencias en un proceso continuo de aprendizaje y reconocimiento del contexto y la cultura en la que se desenvuelven; por esta razón, las respuestas e interpretaciones de una experiencia son únicas y se ejecutan en función del bagaje adquirido y cuya adquisición puede realizarse de un modo consciente o inconsciente (Eric R, James h, \& Thomas M, 1997) Con relación a este planteamiento, Schmitt (1999), habla de la existencia de cinco clases de marketing experiencial, dentro de los cuales se encuentran el marketing de sensaciones y el marketing de sentimientos que conforman el denominado marketing emocional; entendiendo por Marketing de Sensaciones aquel cuya finalidad es proporcionar un placer estético, emoción, belleza y satisfacción por medio de la estimulación sensorial.

Para lograr un impacto sensorial se establece el modelo EPC (estímulo, proceso y consecuencia). El estímulo es la decisión de prestar atención a la información recibida y mantener o guardar la información sensorial, siendo más eficaces aquellos estímulos relacionados con situaciones vividas o significativas. El proceso hace referencia a cómo se lleva a cabo la estimulación, existiendo diversas modalidades para transmitir la información (visual, auditiva, olfativa y táctil). En este marco, el Marketing Sensorial o de Sensaciones se constituye como un elemento diferenciador al atraer al cliente utilizando la estimulación de los sentidos. (Schmitt, 1999)

El marketing de emociones ha tomado auge porque después de varias investigaciones se ha podido comprobar que la comunicación entre consumidores y productos dejo de ser tan lógica y empezó a ser más emocional y efectivamente funciona, porque la emoción domina la razón, lo difícil es que las emociones no se pueden expresar verbalmente, entonces el consumidor conscientemente nunca dirá que es lo que realmente quiere, y algunas marcas o empresas fracasan porque no entienden este paradigma, porque el consumidor siempre miente, no porque quiera sino porque el cerebro córtex, que es el encargado de la parte lógica, es incapaz de verbalizar los instintos y las emociones" (Karmarkar, 2011)

Por eso, las estrategias que están enfocadas en los pensamientos racionales ya no funcionan, dado que el consumidor ya no busca atributos sino experiencias, y desarrollar experiencias de consumo es muy poderoso, pues todo aquello que emociona tiende a ser más recordado que aquello que no emociona, una mala emoción tendrá un mal recuerdo y de la misma manera pasa con las emociones positivas, tendrán un recuerdo positivo, en este sentido, el marketing emocional es esencial para entregar valor y crear lealtad del cliente, lo cual es necesario para los beneficios económicos y de expansión de cualquier empre- 
sa. (Caro J \& Piñero, 2014). Por lo cual, se considera que los clientes pueden estar satisfechos con un servicio y las empresas deben saber como mantenerlos con un proceso interactivo que estimule los recuerdos positivos vinculados con el uso del bien o servicio que la organización ofrece (Terra, Torres, \& Chauvel, 2013)

Por lo anterior, es importante entender que el mercadeo emocional está pensado desde tres sistemas: el biológico, el físico y el químico, y todos tienen impacto sobre sus actividades y dimensiones, de espacio, tiempo e identidad, las cuales permiten observar dichas prácticas desde diferentes lógicas, que se conocen como 'códigos simbólicos', los cuales ayudan a crear significados dentro de la cultura, y esto es muy relevante porque "las cosas no valen hasta que no se les da un significado" (Rapaille, 2007)

\section{METODOLOGÍA}

Se trata de una investigación cualitativa de tipo hermenéutico, que tiene como unidad de análisis el sector funerario de Manizales y Armenia (Colombia). Los datos fueron obtenidos por medio de entrevistas en profundidad y observación; su procesamiento se hizo con el apoyo del Software Atlas Ti. Se desarrolló una exploración, descripción y análisis crítico de los datos recolectados, a partir de ellos se logra la comprensión de la relación entre el marketing emocional y las estrategias de mercadeo en la industria funeraria.

Para este efecto se usan fuentes primarias y secundarias, las fuentes primarias, son aquellas que proveen un testimonio o evidencia directa sobre el tema de investigación. Las entrevistas en profundidad se aplicaron a directores comerciales de organizaciones Funerarias con cobertura nacional pero que tienen sedes en las ciudades colombianas de Armenia y Manizales, también fueron entrevistados algunos asesores de venta de las mismas organizaciones.

El proceso de observación se realizó en dos momentos diferentes de prestación del servicio; el primer momento observado fue en la venta de los planes exequiales, dentro de las instalaciones de la empresa, buscando identificar la forma como se lleva a cabo el contacto de los empleados con los posibles clientes, la argumentación usada en la venta y el tipo de promesas de venta; la información recolectada y el manejo de la temática frente a la emocionalidad de las personas; las respuestas obtenidas en los servicios post-venta frente a los requerimientos o las inconformidades de los clientes, esto fue contrastado con lo observado en las entrevistas a de los directivos y agentes comerciales.

El segundo momento de observación, se hizo cuando la organización debió activar el protocolo de servicio, buscando verificar el cumplimiento de la promesa de venta, que ocurre cuando se requiere la efectiva prestación del servicio; detectando cual es el acompañamiento por parte de la empresa al beneficiario y las sensaciones percibidas de los usuarios y de esta forma determinar si se aplican los planteamientos del marketing experiencial y si se cumple con lo que está planteado en las entrevistas.

\section{ANÁLISIS DE RESULTADOS}

Figura 1: Relación Estrategia - Emocionalidad

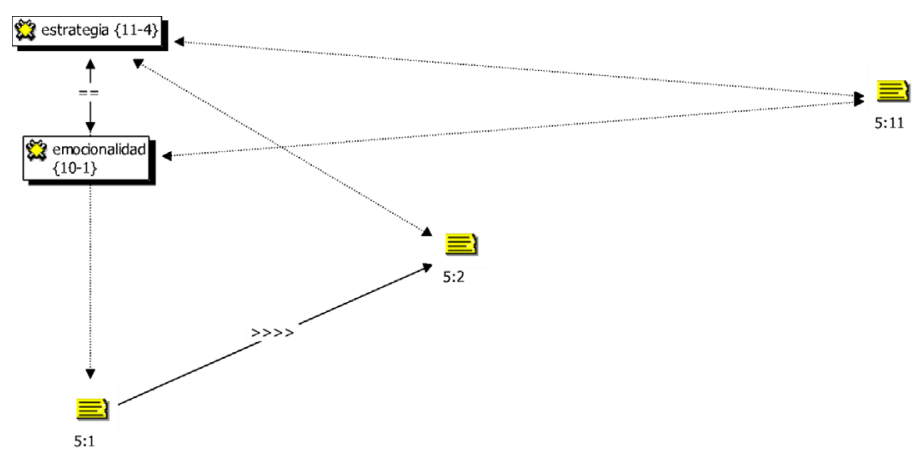

Fuente: Elaboración propia con base en resultados de ATLAS TI

Los recuadros de mayor tamaño corresponden a las categorías entre las cuales se establece una relación y los recuadros pequeños a las citas de las entrevistas que relacionan dichas categorías, donde el primer número corresponde al número de la 
entrevista y el segundo a la cita; esto mismo aplica para todas las figuras; en el caso de la figura 1, se puede observar la correspondencia existente entre las categorías estrategia y emocionalidad como hallazgos en las entrevistas realizadas en el estudio; con ayuda del programa ATLASTI se realizaron las correlaciones de las diferentes respuestas obtenidas de los entrevistados.

Con base en la información recolectada se observa que, las empresas hacen uso del impacto que generan los mensajes transmitidos para estimular emociones en las personas, el caso de la industria funeraria no es la excepcion cuando empieza a desarrollar actividades postmortem como los talleres de duelo, los cuales se usan como estrategia de tipo emocional, para crear lealtad en sus clientes actuales y captar nuevos clientes, Un Directivo consultado se refiere a ello planteando:

Nosotros manejamos una programación bimensual que ya está por todo el año y con quince o veinte días de anticipación empezamos a llamar a las personas, generalmente a los que han tenido pérdidas de seres queridos durante el periodo, los invitamos, generalmente van con la familia, con el hermano, con el esposo, el papá; los talleres han sido un éxito, esperamos cuarenta personas y aparecen ochenta, esperamos cincuenta y llegan cien, empezamos a las seis de la tarde para terminar a las ocho y se va hasta las nueve y media o diez de la noche, porque la gente se queda ahi'”(E4.5-11).

\section{Y complementa:}

Hay algo adicional, tenemos un psicólogo que se ha conectado mucho con esa parte de la emocionalidad; entonces, ha sabido llevar muy bien, pautar, guiar, tiene mucho conocimiento; de hecho, es especializado en duelo en España, él sabe llevarlo muy bien, entonces ha sido súper agradable, la gente sale súper contenta porque él hace todo el proceso de llevarlos hasta el punto de quiebre y después se hace el proceso de reconectar a la persona." (E4.5 - 12).

De esta manera, la empresa aprovecha la condición de sensibilidad en que se encuentran las personas para prestar un servicio que no está incluido en la promesa de venta inicial, ni ha sido pactado en el precio, pero que dada la condición de emocionalidad en que se encuentra el consumidor, contribuye a generar conexión con la empresa y con la marca, lo que a futuro se debe reflejar en la satisfacción del consumidor y elevar la probabilidad de repetición de la compra.

Desde la teoria del marketing emocional, este tipo de iniciativas resulta positiva para la imagen de la empresa, pues logra entender el papel de la emocionalidad en la toma de decisiones como lo plantean Moral y Hernandez (2011): "el papel de las emociones como elemento clave del comportamiento de los consumidores, cuestionando de este modo, que se trate de un proceso de decisiones basado exclusivamente en una serie de argumentos racionales, afirmándose la idoneidad de analizar y estudiar las experiencias que el consumidor desarrolla con la compra y uso del producto".(P.240)

En este sentido debe tenerse en cuenta la relación observada entre las categorías servicio y estrategia, en las que los entrevistados describen cuales son las estrategias que implantan en sus empresas para mejorar las experiencias que el consumidor desarrolla al hacer uso de un servicio. Las empresas funerarias entrevistadas plantean estar un paso delante de la muerte en cuanto al ofrecimiento y prestación de sus servicios, de allí se deriva el nombre de previsión exequial para uno de sus productos; lo que requiere una adecuada organización por parte de la empresa para prestar un buen servicio al cliente, materializado en una buena experiencia de consumo; por tanto, el conocimiento de las emociones de las personas, se convierte en un factor fundamental en la definición de la estrategia orientada a la satisfacción del cliente con el servicio recibido.

Desde la perspectiva de las prácticas se puede observar lo planteado por uno de los ejecutivos comerciales entrevistados:

Nosotros somos una compañía dedicada a satisfacer de manera anticipada la necesidad en el momento de dolor de una persona, usted sabe que cuando una persona tiene un fallecimiento lo que menos necesita es más estrés, entonces ¿qué 
es lo que ofrecemos? tranquilidad en ese momento tan duro de la vida, que con una sola llamada atendamos ese servicio y ellos se despreocupen absolutamente de todo, entonces más que todo esa es como la misión que tenemos y lo que hacemos con las personas" (E 2: 1)

Sin embargo, la calidad del servicio prestado puede ser evaluada de manera diametralmente opuesta por cada una de las personas, el prestador puede encontrarla muy buena y el beneficiario muy mala. (Eigler \& Langear, 1989); en esta perspectiva, uno de los ejecutivos de una organización consultado expone:

Después de cada servicio uno o siente la satisfacción de que le digan muchas gracias, nos sentimos muy bien, nos sentimos como en casa, pero en general son más las críticas que se reciben, como la cuestión de los baños no esté en perfecto estado, que falta una cosa, que falta un bombillo, que falta una toalla para secarse las manos, que el tinto no estaba bien, que no se sintieron acompañados que en ningún momento el coordinador se apareció por allá para por lo menos presentarse y decir buenas noches o buenos días, yo soy quien los va a acompañar, lo que necesiten... o sea ponerse uno en servicio de la gente, porque ahí es que uno tiene que atender a todas las personas que lleguen, sean dolientes, amigos, los que sean a la hora que sea. (E3: 5)

La percepcion de calidad tiene realacion entre las condiciones psicologicas y emocionales que intervengan en el momento de la acción. asi mismo, Eigler \& Langear (1989) afirman: "un mismo servicio, producido por las mismas personas, no presenta automaticamente una calidad estable en el tiempo". (p.7). Igualmente, se observa la relación entre las acciones que emprenden las empresas del sector funerario al ofrecer el servicio, especialmente en el inicio del mismo, pues es allí donde emergen las emociones de los clientes, están expuestos todos sus sentimientos y la relación existente entre el funcionario de la empresa y el beneficiario es la que humaniza o le quita el sentido humanizante a toda la actividad comercial.

En este sentido, es necesario tener en cuenta que prestación de servicios de la industria funeraria tiene características algo complejas, en cuanto a la relación de los agentes que intervienen en la creación, prestación y uso del servicio, si el output del sistema está siempre constituido por un servicio, otros tres elementos son necesarios para su fabricación: dos personas y un producto. Cuando se ofrece un servicio exequial se prometen muchos beneficios dentro del plan funerario como lo son: el cofre, arreglo floral, traslado del cuerpo, sala de velación entre otros; en las entrevistas con relación al proceso del prestación del servicio se observó:

La persona llama, nosotros vamos le recogemos el cuerpo, lo llevamos a tanatopraxia, que es un tratamiento estético del cuerpo, no es una autopsia, ni necropsia, nosotros no somos dictaminadores de causas de muerte, nosotros simplemente lo que hacemos es embellecer el cuerpo, de ahí pasamos a que la persona escoja el cofre donde va a ser depositado el cadáver, hace un selección en una sala que nosotros tenemos, después de que han elegido, nosotros introducimos el cuerpo al ataúd y lo llevamos a una sala que llamamos la sala de liberación, es un espacio donde tenemos contacto única y exclusivamente con la familia antes de sacarlo a sala de velación, ellos aprueban el cuerpo, así en las condiciones que esta, hay muchas veces que lo devuelven que mire que las uñas las tiene sucias, hay veces hasta que mire que por favor me le pinte el cabello, que me le suba al rubor que me le suba tal cosa, y ese tipo de cosas, de la presentación cámbiemele el vestido todo eso es la aprobación con ellos y adicionalmente se hace un momento de oración con una persona que tenemos acá especializada en orar y de acuerdo al credo que la persona profese pues lo hacemos, acá estamos preparados para recibir católicos, evangélicos, cristianos etc., entregan unas velas, ellos mismo se encargan de apagar la luz, esa luz que se está yendo pero hay que encender de nuevo otra, es como una especia de ritual de despedida, pero todo parte del respeto, antes de un paso a seguir le contamos a la familia y si la familia no quiere hacer el ritual se pasa directamente a la sala de velación. (E4.5:17)

Como puede verse en este relato, se trata de crear una experiencia de servicio alrededor de una situación compleja, que va más allá de las tradicionales condolencias o mensajes que pue- 
den tener diversas interpretaciones por parte del consumidor, pero con el tipo de ritual, donde se busca satisfacer al cliente se le está dando una mayor importancia, diciéndole de manera indirecta que es valioso para la organización, por lo cual, ella está dispuesta a ofrecerle diversos satisfactores por la medio de la atención a su ser querido, esto podría constituirse en un factor diferenciador logrado por medio de marketing experiencial.

Durante la observación de la prestación del servicio, se buscó evidenciar la coherencia entre lo planteado por las empresas funerarias en el proceso de entrevistas y la forma en que se presta el servicio, si las prácticas corresponden con lo que se dice. Sin embargo, se encontró que a la hora de prestar un servicio, muchos de los ítems que hacen parte de la promesa de venta no se cumplen, como el acompañamiento por parte de la empresa a la hora de la escogencia de cofres, las condiciones estéticas del cuerpo antes de su presentación en sala de velación, arreglos florales, ritual espiritual, oraciones y el estar pendiente de los dolientes durante todo el proceso hasta la cremación o inhumación, de esta manera aunque las empresas conocen las técnicas del marketing experiencial, al parecer no hacen uso adecuado de ellas para crear lealtad en el cliente.

Son muchos los factores o detalles que intervienen a la hora de recibir y prestar el servicio, factores que no son lo suficientemente claros en el momento que se está ofreciendo el plan exequial, dado que los asesores de ventas no amplían la información lo suficiente al usuario; de allí que cuando se presenta el evento las personas tienen expectativas totalmente diferentes a la realidad del servicio ofrecido. Entonces advierten la necesidad de productos que creían estar incluidos en el plan adquirido, pero como la necesidad esta generada y el servicio no está disponible deben asumir costos adicionales, lo que genera insatisfacción del cliente borrando todos los esfuerzos que se hagan en la perspectiva del marketing emocional, esto se evidencia en lo que afirma uno de los directivos comerciales:

Si, de hecho mucha gente empieza a llamar que mire que el ramo yo lo quiero blanco y me trajeron uno amarillo, por decir algo, entonces el asesor tiene que estar pendiente de todas esas cositas, todos esos detallitos de ese servicio, no todos los servicios son iguales, hay gente que solo vienen cuatro personas $y$ ese es todo el servicio, como hay gente que eso es impresionante porque hay que sacar sillas, gente en la calle porque no caben, entonces todo depende, pero todos los servicios que no están dentro del paquete generan un costo adicional (E2:6)

A partir de esto se puede decir que la humanización del servicio depende del factor dinero olvidando el factor humano, o la aplicación de herramientas del marketing emocional, sin considerar lo que Vélez (2006) señala al afirmar que la empresa funeraria debe tener una doble sensibilidad frente al concepto de ser humano, es decir, como organización su constitución y esencia son las personas que la conforman, sin ellas la organización no existiría y, además, su papel social es la manipulación del cadáver, la vivencia de la muerte en la cultura. La empresa funeraria presencia la muerte de los otros bajo una perspectiva económica, lo que la puede tentar a percibir que recibe un objeto, un insumo, una mercancía, cegando el reconocimiento del otro, ya cadáver, en su calidad de sujeto y, por tanto, merecedor de un trato digno, o desconociendo la situación emocional del otro en su condición de doliente.

También se observa que las empresas del sector funerario, no están haciendo uso de la humanización del servicio como una estrategia durante las diversas etapas de contacto con el cliente, sino, como un enganche de venta después del fallecimiento del ser querido, sin tener en cuenta que en ambas situaciones las percepciones son diferentes y por lo tanto requieren un manejo distinto, uno de los directivos entrevistados señala:

Hay personas que se trabajan en frio y hay personas que se trabajan en caliente, las personas en frio son las que no tienen necesidad inmediata en el momento de la compra, pues lo compran para un futuro evento que no sabe cuándo va a ocurrir; el trabajo en caliente es cuando a la persona le acaba de fallecer un familiar, entonces esa es una persona caliente, esas personas se manejan muy diferente porque son muy susceptibles, es la persona que más dispuesta a todo lo que uno le ofrezca (E2:4)

De otro lado, también se observó que las empresas no tienen 
en cuenta a los beneficiarios durante el suceso, hace falta acompañamiento y el estar atento a las necesidades que surjan, pues allí, se efectúan las relaciones que dan lugar a la calificación del servicio, tal y como lo manifiestan los directivos de estas empresas, es difícil el manejo de las emociones en esa circunstancia, pero es en ese preciso momento cuando se debe tener mayor control para que el cliente se sienta respaldado y fortalecido emocionalmente, que no se sienta solo y que encuentre en la empresa un proveedor incondicional ante sus necesidades, de tal manera que se pueda establecer un vínculo entre el estado emocional del cliente y el servicio que ofrece la empresa.

Se observó una constante en la prestación del servicio post-mortem en las compañías indagadas que usan estrategias de venta y fidelización; tal y como los autores analizan a lo largo de sus trabajos, se empiezan a incorporar en el pensamiento estratégico variables sociopolíticas y culturales relacionadas con la empresa, dejando de lado no solo la mirada estática de la estrategia, sino también desplazando los análisis meramente cuantitativos. (López P. A., 2013); En este sentido, uno de los gerentes entrevistados señala:

$\mathrm{Al}$ crear emociones más positivas en la gente, digamos que hay una parte que se tomó de la Europa Oriental donde las personas lo que hacen es una fiesta cuando hay una muerte, cuando hay un fallecimiento, pero no una fiesta en el sentido de carnaval y todas esas cosas, sino de una reunión, de compartir entre familiares y hacer un homenaje a esa persona, o sea evidenciar esos momentos buenos de esa persona, festejar y dar gracias por haberlo tenido ese tiempo acá, eso es lo que pasa ahora, desde ahí se creó todo un cuento, a partir del cual se generaron unos arquetipos de una batalla de todas las cosas que nosotros libramos, para conectarnos con las emociones de todas las personas y brindarles algo nuevo a partir de eso, es realmente un homenaje al amor. (E4.5: 3-4)

Esta estrategia empleada por esta Empresa del Sector Funerario resultaría positiva para los familiares del difunto, pues como lo afirma (Vélez, 2006) Más allá de la presencia de un cadáver que muestra el fin de una vida, quedan los que sobreviven a esa muerte y un sin número de interrogantes que surgen a partir de un proceso de duelo y despedida, esto constituye uno de los elementos fundamentales del marketing emocional.

No obstante, el cliente responde tanto a estímulos positivos como negativos, el hecho de que el prestador del servicio exponga al cliente a situaciones en las cuales exista una confrontación entre lo que se le ofreció inicialmente y lo que realmente está recibiendo, pero si ésta resulta lejana a una sensación de satisfacción con el servicio prestado, genera desequilibrio en el sistema por una serie de inconformidades e impresiones de engaño (falta de claridad en la información), tal como lo expone uno de los entrevistados:

Que el servicio es muy caro y es de muy baja calidad, las personas se sienten engañadas y por lo general mucha inconformidad de parte de los usuarios, que el ramo no les gusto, que el cofre estaba pelado, que la carroza es muy fea, que el transporte fue muy poco, no contrataron los buses que debían ser entonces que la gente quedo o muy apeñuscada o hubo gente que no pudo asistir al evento como tal porque la empresa dice muchas cosas a la hora de afiliar a las personas pero los engañan y cuando ya tienen que utilizar el servicio les salen con cosas totalmente diferentes" (E3:6)

\section{CONCLUSIONES}

La aplicación de las estrategias del marketing relacional o del marketing emocional en las empresas del sector funerario, requiere de una activa participación de las personas, por lo cual en el proceso de humanización del servicio contar con personal calificado y con la personalidad adecuada para enfrentar los retos que estas estrategias imponen, se convierte en un factor crítico de éxito para este tipo de organizaciones.

Por lo anterior las empresas del sector funerario deben mostrar una real vocación de trabajo sobre el componente de calidad de vida laboral, con el fin de contar con el personal calificado y con la disposición emocional que este tipo de trabajos demanda, con lo cual se debe llegar a mejorar los niveles de estabilidad en los cargos, especialmente de las personas que tienen contacto directo con los clientes. 
La comunicación interna, debe ser otro factor clave para el éxito de la estrategia empresarial, de tal forma que se dé un trabajo continuo por parte de la gerencia en la generación de un alto nivel de pertenencia entre los trabajadores y que estos comprendan que además de una actividad económica su trabajo también tiene un alto componente de beneficio social y que los clientes que atienden se encuentran en una situación diferente a los clientes de cualquier otro negocio, esto se verá reflejado en el servicio.

De esta manera se puede lograr que las experiencias del cliente como resultado de los estímulos provocados en los sentidos y en la mente del cliente durante las situaciones vividas por la prestación del servicio permitan una adecuada conexión entre la empresa, la marca y los clientes; de tal manera que la experiencia que en principio por la situación del cliente puede ser negativa, gracias a los valores emocionales, cognitivos o sensoriales que el cliente obtenga, cambien, para convertirse en un buen recuerdo por parte del cliente con respecto a la organización, llevando a una satisfacción del cliente que permita una buena relación de este con la empresa; lo que seguramente redundará en nuevas compras. (Estebana, Delgado B, \& Pelaez M, 2014)

En la perspectiva del marketing emocional se plantea la necesidad comprender el cliente como un individuo no solamente racional sino como un sujeto que desea recibir estímulos y experiencias cargadas de emotividad y creatividad en su relación con el producto y la empresa; sin embargo, pocas de las empresas observadas, implementan esa combinación exitosa de innovación y emocionalidad.

No obstante, también se observaron empresas que han visto en el marketing experiencial y emocional una alternativa amplia y poco explorada por la competencia, entendiendo las emociones como factor clave en sus procesos comerciales y de mercadeo; lo que las ha llevado a implementar estrategias basadas en uso de colores y luces en sus instalaciones, talleres de duelo, adecuaciones de la planta física que permitan una mayor comodidad a las personas que usan sus servicios, y otros elementos que permitan un manejo de la emocionalidad de los clientes.
La humanización del servicio en la industria funeraria, es una de las estrategias que plantean muchos de los entrevistados, teniendo en cuenta que la muerte de un ser significa para sus familiares la pérdida de una parte importante de sus vidas, la terminación de un legado familiar; y para la empresa funeraria ese ser es un cadáver que lo conecta con sus futuros demandantes, y para lo cual se dispone a prestar un servicio.

En la perspectiva de la humanización del servicio, es necesario innovar en las estrategias de acompañamiento y atención a las necesidades que surjan durante la prestación del servicio, pues allí, se materializan las relaciones que dan lugar a la calificación del servicio, tal y como lo manifiestan los directivos de estas empresas, es difícil el manejo de las emociones en esa circunstancia, pero es en ese preciso momento cuando se debe tener mayor control para que el cliente sienta el respaldo de la organización que convierta la situación en una emoción positiva frente a la empresa, de tal manera que se pueda establecer un vínculo entre el estado emocional del cliente y el servicio que ofrece la empresa.

Es necesario adoptar medida para cuidar los detalles que intervienen en la prestación del servicio y que estos correspondan con la promesa de venta hecha al ofrecer el plan exequial, de tal forma que los asesores de ventas provean la información suficiente sobre las características del producto, los derechos y deberes de los usuarios; de tal manera que cuando llegue el momento de prestar el servicio, las expectativas de los clientes coincidan con el servicio ofrecido, lo que genera satisfacción del cliente.

Se trata de crear una experiencia de servicio alrededor de una situación compleja, en la que las estrategias de marketing experiencial van más allá de las tradicionales condolencias o mensajes que pueden tener diversas interpretaciones por parte del consumidor, sino que los rituales que se establezcan se llegue a satisfacer al cliente, dándole mayor importancia, haciéndole sentir que es valioso para la organización, por lo cual, ella está dispuesta a ofrecerle diversos satisfactores por la medio de la 
atención a su ser querido, esto podría constituirse en un factor diferenciador logrado por medio de marketing experiencial.

\section{REFERENCIAS}

Barrios, M. (2012). Marketing de la Experiencia: principales conceptos y características. Palermo Business Review. No 7, 67 $-89$.

Caridad, M., Castellano, M. I., \& Hernández, M. F. (2015). Marketing Experiencial para la Promoción del Proceso Educativo a Nivel de Pregrado: Un estudio de dos Universidades privadas. Revista Facultad de Ciencias Económicas. Vol. 23. No 2, $81-95$.

Caro J, M. C., \& Piñeros, M. S. (2014). Las emociones y la resistencia al cambio de las actitudes. Revista Española de Investigación de Marketing ESIC. No 18, 17 - 31

Casani, F., Rodríguez-Pomeda, J., \& Sánchez, F. (2012). Los Nuevos Modelos de Negocio en la Economía Creativa: Emociones y Redes Sociales. Universia Business Review, 48-68.

Clarkson, J. J., Tormala, Z. L., \& Rucker, D. D. (2011). Cognitive and affective matching effects in persuasion: An amplification perspective. Personality and Social Psychology Bulletin, No 37, 1415-1427.

Eigler, P., \& Langear, E. (1989). Servucción: El marketing de Servicios. Madrid: McGraw Hill.

Eric R, K., James h, S., \& Thomas M, J. (1997). Neurociencia y Conducta. Madrid: Prentice Hall.

Estebana, A. A., Delgado B, E., \& Peláez M, J. (2014). ¿Quién ama a las marcas? Determinantes personales y de consumo. Revista Española de Investigación de Marketing ESIC, 2 - 16.

García, L. (2010). Marketing Experiencial en Sectores de Servicios con Establecimientos de Atención al Público. Madrid: Universidad Complutense de Madrid.
Hirschman, E. (1999). The Roles of Emotion in Consumer Research. Advances in consumer research, 4-11.

Holbrook, M. B., \& Hirschman, E. C. (1982). The Experiential Aspects Of Consumptiom: Consumer Fantasies, Feelings and Fun. The Journal of Consumer Research, Vol.9, No 2, 132 - 140.

Jiménez B, J., \& Martinez C, S. (2016). Marketing Experiencial en FITUR: Análisis de dos destinos competidores, Islas Canarias e Islas Baleares. Revista de Turismo y Patrimonio Cultural. Vol. 14, No. 1, 75 - 91.

Karmarkar, U. R. (2011). Note on Neuromarketing. Boston: Harvard Business School.

Klaric, J. (2012). Estamos ciegos. Lima: Planeta.

López, B. (2006). Marketing y emociones. Business \& Marketing, 23-39.

López, P. A. (2013). Una aproximación al campo de estudio del pensamiento estratégico desde las publicaciones académicas: de lo predecible a lo emergente. Investigación y Reflexión, vol. XXI. N. 1, 217 - 241.

Marci, C. (2008). Minding the gap: the evolving relationship between affective neuoscience and advertising research. intenational journal of advertising, 474-491.

Moral, M., \& Fernández, M. T. (2011). Nuevas Tendencias del Marketing: El Marketing Experiencial. Entelequia, 237-251.

Pine, B., \& Gilmore, J. (1998) The Experience Economy: work is theatre and every business a stage. Boston: Editorial Harvard Business School Press.

Rapaille, C. (2007). Código Cultural. México: Norma.

Schmitt, B. H. (1999). Experiential Marketing: How to Get Customers to Sense, Feel, Think, Act, and Relate to Your Company and Brands. New York: New York: Free Press. 
Terra, L., Torres, A., \& Chauvel, M. (2013). As Práticas de marketing, entre transações e Relacionamentos, de empresas no Brasil. Brazilian Business Review. Vol. 10. No 2, 124-141.

Winterich, K. P., \& Haws, K. L. (2011). Helpful Hopefulness:
The effect of future positive emotions on consuption. Journal of Consumer Research. No 38, Vol. 3, 505-524.

Vélez, C. (2006). Hacia una humanización de la empresa funeraria. Pensamiento y gestión, No. 21, 93 - 116. 